\title{
Toma de decisiones rápidas: investigación de la relación entre múltiples tareas e impulsividad
}

\author{
Making quick decisions: an investigation on the relationship between multiple tasks \\ and impulsiveness
}

Tomada de decisões rápidas: investigação da relação entre múltiplas tarefas e impulsividade

\author{
Valéria Marcondes Pereira, Doctora \\ Academia de la Fuerza Aérea - AFA \\ Pirassununga/SP - Brasil \\ valeria_afa@yahoo.com.br
}

Luiz Maurício de Andrade da Silva, Doctor Academia de la Fuerza Aérea - AFA Pirassununga/SP - Brasil Ima28@uol.com.br

\section{RESUMEN}

Este artículo forma parte de un proyecto de investigación que tiene el objetivo de contribuir con el entrenamiento de pilotos militares, específicamente con el entrenamiento para decisiones rápidas. El punto de partida de la investigación es la prueba de aptitud para pilotaje militar (TAPMIL), que evalúa el potencial de aprendizaje del candidato para el pilotaje militar, con el objetivo de instrucción de vuelo realizada en los Escuadrones de Instrucción Aérea de la Academia de la Fuerza Aérea (AFA). Es un una prueba psicológica totalmente informatizada, compuesta por una batería de seis testes que evalúan, de manera aislada o combinada, aptitudes inherentes al pilotaje, como "capacidades cognitivas" (por ejemplo, procesamiento de información, velocidad y precisión de respuestas, memoria de trabajo) y capacidad en "tareas múltiples" (psicomotoras y cognitivas combinadas) (COSTA, 2010, p. 6). Se buscan evidencias para atribuir pesos a las diferentes variables intervinientes en el problema de la aptitud (SILVA et al., 2009), lo que nos remite a elegir - hasta el presente momento - como variable de destaque, la capacidad de ejecución de múltiples tareas. El foco, en la variable 'múltiples tareas', nos llevó a nuevas líneas investigativas, como estudios sobre el juicio humano en decisiones, sobre todo aquellas tomadas en ambientes dinámicos y de gran incertidumbre (KLEIN, 2009), y la correlación existente entre la capacidad de ejecutar múltiples tareas y la impulsividad. Pretendiendo continuar esa línea investigativa a respecto de la correlación existente entre múltiples tareas e impulsividad, el presente trabajo tiene como objetivo presentar los resultados de dos testes realizados con los cadetes de la Fuerza Aérea Brasileña, aviadores del grupo de 2011, en un total de 60 voluntarios. Para verificar la capacidad de ejecutar múltiples tareas, se utilizó la prueba "todo al mismo tiempo" (HERCULANO-HOUZEL, 2014). Para la prueba de impulsividad, con 45 (cuarenta y cinco) voluntarios, se usó la Escala de Impulsividad de Barratt - BIS 11.

Palabras clave: Múltiples tareas. Impulsividad. Tomada de decisión. Desempeño.

Recibido / Received / Recebido 22/09/14
Aceptado / Accepted / Aceito 22/05/15 


\section{ABSTRACT}

This article is part of a research project that aims at contributing to the training of military pilots, specifically training for quick decisions. The starting point of the research is the aptitude test for military flying (TAPMIL), which assesses the candidate's learning potential for military flying, with a view to flight instruction performed in the Air Instruction Squadrons of the Air Force Academy (AFA). It is a fully computerized psychological test, comprised of a battery of six tests that assess, either alone or in a combined manner, inherent flying skills, such as the "cognitive capacities" (e.g., information processing, speed and accuracy of responses, working memory) and the ability to "multitask" (psychomotor and cognitive combined) (COSTA, 2010, p. 6). Evidence are sought for assigning weights to the different variables intervening in the aptitude problem (SILVA et al., 2009), which brings us to choose - to date - as a featured variable, the ability to run multiple tasks. The focus in the "multitask" variable led us to new investigative lines, such as studies on human judgment in decisions, particularly those taken in dynamic environments and of great uncertainty (KLEIN, 2009), and the correlation between the ability to perform multiple tasks and impulsiveness. In order to continue this investigative line about the correlation between multiple tasks and impulsiveness, this study aims at presenting the results of two tests performed with the Brazilian Air Force cadets, aviators from the class of 2011, a total of 60 (sixty) volunteers. To verify the ability to multitask, the "all at once" test was used (HERCULANO-HOUZEL, 2014). For the impulsiveness test, 45 (fortyfive) volunteers used the Barratt Impulsiveness Scale - BIS 11.

Keywords: Multitasking. Impulsiveness. Decision-making. Performance.

\section{RESUMO}

Este artigo é parte de um projeto de pesquisa que tem o objetivo de contribuir com o treinamento de pilotos militares, especificamente com o treinamento para decisões rápidas. O ponto de partida da pesquisa é o teste de aptidão para pilotagem militar (TAPMIL), que avalia o potencial de aprendizagem do candidato para a pilotagem militar, com vistas à instrução de voo realizada nos Esquadrões de Instrução Aérea da Academia da Força Aérea (AFA). É um teste psicológico totalmente informatizado, composto por uma bateria de seis testes que avaliam, de maneira isolada ou combinada, aptidões inerentes à pilotagem, como "capacidades cognitivas" (por exemplo, processamento de informação, velocidade e precisão de respostas, memória de trabalho) e capacidade em "tarefas múltiplas" (psicomotoras e cognitivas combinadas) (COSTA, 2010, p. 6). Buscam-se evidências para a atribuição de pesos para as diferentes variáveis intervenientes no problema da aptidão (SILVA et al., 2009), o que nos remete a eleger - até o presente momento - como variável de destaque, a capacidade em execução de múltiplas tarefas. O foco, na variável 'múltiplas tarefas', levou-nos a novas linhas investigativas, como estudos sobre o julgamento humano em decisões, sobretudo aquelas tomadas em ambientes dinâmicos e de grande incerteza (KLEIN, 2009), e a correlação existente entre a capacidade de executar múltiplas tarefas e a impulsividade. Com vistas a continuar essa linha investigativa a respeito da correlação existente entre múltiplas tarefas e impulsividade, o presente trabalho tem por objetivo apresentar os resultados de dois testes realizados com os cadetes da Força Aérea Brasileira, aviadores da turma de 2011, em um total de 60 voluntários. Para a verificação da capacidade de executar múltiplas tarefas, utilizou-se o teste "tudo ao mesmo tempo" (HERCULANOHOUZEL, 2014). Para o teste da impulsividade, com 45 (quarenta e cinco) voluntários, utilizou-se a Escala de Impulsividade de Barratt - BIS 11.

Palavras-chave: Múltiplas tarefas. Impulsividade. Tomada de decisão. Desempenho.

\section{INTRODUCCIÓN}

El hombre, como ser social, es complejo en su totalidad. Hay influencias en la relación entre individuo y organización, siendo que el administrador debe tener en cuenta el análisis del individuo en particular y en la colectividad del ambiente organizacional.

La desinhibición comportamental constituye una incapacidad de control personal en respuesta a exigencias de la situación. El fracaso, en la inhibición de comportamientos, ha recibido en la literatura la denominación de impulsividad.
El propósito de este trabajo es investigar la relación entre la ejecución de múltiples tareas y la impulsividad. Hay relatos en la literatura de que aquellos que se juzgan más aptos para ejecutar múltiples tareas son exactamente los más impulsivos (SABONMATSU et al., 2013). Así, se pretende verificar si lo mismo ocurre en una muestra de cadetes de la Academia de la Fuerza Aérea Brasileña.

Estudios de comportamiento humano, bajo la mirada de la neurociencia, parten del siguiente principio: "lo que somos, hacemos, pensamos y deseamos es resultado del funcionamiento del sistema nervioso y su interacción con el cuerpo". La neurociencia procura entender la 
relación de la estructura y del funcionamiento del sistema nervioso con la historia de vida de cada uno; la cultura, la sociedad, y la genética hacen de nosotros lo que somos, individualmente, como seres humanos, y como animales (HERCULANO-HOUZEL, 2014).

\section{REFERENCIAL TEÓRICO}

El referencial teórico se desarrolla bajo los temas de la cognición organizacional y de las decisiones rápidas.

\subsection{Cognición organizacional}

En lo que atañe a la cognición, hay una pluralidad de lenguajes y conceptos que impregnan ese campo de investigación. Entre las vertientes presentadas en los estudios de Davel y Vergara (2001), se tiene la primera de ellas con la exposición confirmatoria del rompimiento de la noción de mente cartesiana, o sea, la mente ser totalmente separada del cuerpo físico. Eso significa romper con la dicotomía razón y emoción y pasar a visualizar el hombre como un todo, vincular los procesos cognitivos y emocionales al cuerpo, siendo estos considerados centrales para la racionalidad.

Rotulada de constructivista, la segunda vertiente tiene al hombre como sujeto de la historia, y no solo un espectador. Enunciado por Davel y Vergara (2001), la mente como instrumento activo en la construcción del mundo se configura un área específica de investigación en la psicología social, según la cual la naturaleza social de los procesos de cognición van más allá del simple procesamiento de informaciones.
Las organizaciones burocráticas se consolidan con la perspectiva macroorientada en los estudios organizacionales, fundamentados en la sociología, en la ciencia política y en la economía. Según Hall,

La mirada macro en los estudios organizacionales parte del comportamiento de las personas se debe a factores organizacionales. Las organizaciones tienen el poder de moldar el comportamiento y las acciones individuales. Ellas actúan, tienen políticas, hacen declaraciones. Ellas subsisten en el tiempo más allá de las personas que la integran. Hay una estructura social, un sistema de reglas, normas, valores y expectativas que preexisten a los individuos. (HALL, 1984 apud DAVEL; VERGARA, 2001, p. 96, nuestra traduccíon).

Las organizaciones, cuya influencia es de una perspectiva microorientada, son fundamentadas en la psicología. Su raíz está en el movimiento de las relaciones humanas, que trata el concepto de organización como algo más allá de la interacción de individuos. En oposición a la visión anterior, en el papel determinante de la organización sobre los procesos individuales, esa perspectiva enfatiza más el papel del individuo en la construcción de la organización. Según Staw y Sutton,

individuos autónomos posan como organización. Acciones llamadas de organizacionales pueden ser acciones individuales, bajo la apariencia de una entidad impersonal. Individuos con poder ejercen influencia y control: al modelar decisiones estratégicas; al definir estructuras más estables; al modelar percepciones de aliados; al modelar características del propio grupo de personas. (STAW; SUTTON, 1993 apud DAVEL; VERGARA, 2001, p. 96, nuestra traduccíon).

El Cuadro 1 presenta una evolución de los abordajes cognitivistas a partir de los años 90.

Cuadro 1 - Abordajes cognitivistas.

\begin{tabular}{|c|c|}
\hline Autores & Abordajes cognitivistas \\
\hline Wilpert (1995) & $\begin{array}{l}\text { Señala el crecimiento de la perspectiva simbólica y constructivista. } \\
\text { Afirma que es innecesario el conflicto entre los abordajes macro y microorientados. } \\
\text { Aborda el fenómeno organizacional como socialmente construido por medio de la interacción de } \\
\text { autores relevantes. }\end{array}$ \\
\hline Rousseau (1997) & $\begin{array}{l}\text { La organización es vista como "proceso", y no como una "entidad". } \\
\text { Organización como una construcción social. }\end{array}$ \\
\hline $\begin{array}{c}\text { Scneider and } \\
\text { Angelmar (1993) }\end{array}$ & $\begin{array}{l}\text { Articulan tres niveles de análisis (individuo, grupo y organización) y } \\
\text { tres propiedades centrales de la cognición: } \\
\text { - estructuras cognitivas (cómo el conocimiento es adquirido y utilizado); } \\
\text { - procesos cognitivos (cómo el conocimiento es adquirido y utilizado); y } \\
\text { - estilos cognitivos (como las unidades se diferencian en las estructuras y procesos de conocimiento). }\end{array}$ \\
\hline Walsh (1995) & $\begin{array}{l}\text { Los estudios cognitivos se han orientado a cuatro niveles de análisis (individuo, grupo, organización, } \\
\text { industria) y los asuntos cognitivos (representación, uso y desarrollo de las "estructuras de conocimiento"). } \\
\text { El desarrollo y cambios en las estructuras de conocimiento; la representación o la "estructura del } \\
\text { conocimiento" en sí, buscando descubrir, en etapa de estructuras específicas utilizadas por gerentes, sus } \\
\text { atributos, contenidos específicos y como esos se estructuran; y el uso y consecuencias de esas estructuras, } \\
\text { o sea, su impacto sobre resultados organizacionales, en sus diferentes niveles. }\end{array}$ \\
\hline
\end{tabular}

Fuente: Adaptado de Davel y Vergara (2001). 
Entonces, Davel y Vergara (2001) destacan algunas características relevantes sobre el estado del arte en el campo de la cognición y organización.

Los estudios sobre cognición organizacional están orientados a explorar impactos de las estructuras de conocimiento en la vida de las personas, gestores y organizaciones. En contrapartida se encuentra un reducido número de investigaciones involucrando el procesamiento automático, no controlado o inconsciente.

Los abordajes que integran las estructuras cognitivas y procesos de cognición son raros. Y también, es fuerte el presupuesto de que la cognición individual produce comportamiento organizacional que puede conducir a la comprensión de procesos colectivos como sumatorios de procesos individuales. Tal relación requiere justificativa del acto que es destacado después de su ocurrencia.

Cualquiera que sea la perspectiva que consolida la organización, en una vertiente micro o macro, se encuentran tanto individuos, como procesos ocurriendo en la organización. Procesos estos que culminan en lo que se acordó en llamar de decisiones.

\subsection{Tipos de decisiones}

Aunque el arte de la guerra y los movimientos militares hayan influenciado algunos ramos de la investigación teórica respecto de las decisiones, como el de las investigaciones operativas y el de la logística, los principales avances ocurrieron en el ámbito de las organizaciones privadas, de interés económico. Tradicionalmente, tales organizaciones dividen las decisiones en dos grandes bloques: el bloque de las decisiones estratégicas y el bloque de las decisiones operativas y tácticas.

En el bloque de las decisiones estratégicas, el foco es siempre el horizonte temporal más largo y la atención sobre los impactos que tales decisiones pueden producir en la pista de competencia entre las organizaciones. En el bloque de las decisiones operativas y tácticas, el horizonte pasa a ser de plazo más corto y la atención se vuelve a la eficiencia y la productividad de las operaciones.

Con el advenimiento de la economía del comportamiento, nuevos actores pasaron a investigar las decisiones - ahora con mayor foco en el comportamiento humano - bajo el prisma de la psicología cognitiva. En función de eso, las decisiones pasaron a ser analizadas bajo un nuevo prisma (o bloque): (i) las decisiones racionales, tomadas en situaciones planificadas, involucrando apuestas de bajo riesgo, en ambientes de baja presión en términos de tiempo de decisión, y apoyadas principalmente en la matemática y en los postulados de la lógica; y (ii) las decisiones intuitivas, tomadas en situaciones dinámicas, de escasez de tiempo de decisión, y con poco uso del recurso de la deliberación lógica.

En pocas palabras, decisiones estructuradas y decisiones rápidas. Las decisiones rápidas, de carácter más intuitivo, fueron clasificadas como decisiones basadas en el sistema 1 de pensamiento y deliberaciones. Ya las decisiones estructuradas fueron clasificadas como decisiones basadas en el sistema 2 de pensamiento y deliberaciones (BAZERMAN, 2006).

Así, se acordó que las decisiones del tipo 1 serían más dependientes del repertorio de experiencias individuales, que habilitarían el tomador de decisión al mejor uso de su sentido circunstancial y subjetivo, y las decisiones del tipo 2 serían más dependientes del uso de la lógica matemática e instrumental. En el pilotaje militar, dependiendo de cada situación y etapa del vuelo, las decisiones de los pilotos pueden demandar uno u otro sistema, o hasta incluso la conjugación de los dos sistemas de pensamiento y decisión.

En el ámbito del presente trabajo, el interés recae sobre las decisiones más rápidas en ambiente dinámico, como aquel que caracteriza el vuelo. Otra razón para esa iluminación de los asuntos relacionados a decisiones más rápidas e intuitivas se justifica por el tema central de este trabajo, que explora las características de la impulsividad en lo que respecta a la administración de múltiples tareas.

\section{METODOLOGÍA}

Como ya fue dicho, el objetivo de este trabajo es verificar si, en una muestra de cadetes de la Academia de la Fuerza Aérea, la habilidad para administrar múltiples tareas está correlacionada a la impulsividad de esos jóvenes. La investigación contó con un total de 45 (cuarenta y cinco) voluntarios $(\mathrm{N}=45)$.

Para verificar la habilidad en administración de múltiples tareas fueron utilizados dos instrumentos: el software TAPMIL y el juego "todo al mismo tiempo" (TMT) que fue ofrecido por un equipo de neurocientistas coordinados por Suzana Herculano-Houzel. En este juego, cuyo objetivo es obtener mayor número de puntos y, consecuentemente, controlar un mayor número de tareas, se aumentan, gradualmente, las tareas a medida que el jugador cambia de nivel.

Para verificar la impulsividad fue aplicado el instrumento BIS-11, en que se utilizó la Escala de Impulsividad de Barratt - BIS 11. El instrumento consta de 30 (treinta) asuntos, en que el encuestado señala solo una entre las siguientes alternativas: (1) raramente o nunca; (2) de vez en cuando; (3) con frecuencia; (4) casi siempre o siempre. La formatación de los datos siguió la puntuación para las preguntas que van de 1 a $4(1,2,3,4)$. En esas cuestiones 1, 7, 8, 9, 10, 12, 13, 15, 20, 29 y 30, se consideró el orden inverso $(4,3,2,1)$.

Respecto a las diferencias culturales relacionadas a conceptos relativos a los diferentes tipos de impulsividad, el modelo de Barratt - BIS 11 ha sido adaptado con éxito para diferentes culturas, lo que evidencia su validez transcultural. Dada la importancia del modelo propuesto por Barratt - BIS 11 para estudios sobre la impulsividad, se considera que el desarrollo de una versión brasileña de la escala es de fundamental importancia para la práctica clínica y para la realización de estudios sobre el tema (MALLOY-DINIZ et al., 2010, p. 104).

Patton et al. (1995) definen la versión actual del instrumento, realizada por un análisis de componentes principales en BIS-11. Las notas recogidas a partir de una muestra de 248 (doscientos cuarenta y ocho) pacientes siquiátricos internados y 412 (cuatrocientos doce) estudiantes universitarios. El análisis factorial, a partir de esos casos, reveló tres factores de segunda orden, aquí denominados secundarios, y seis factores oblicuos de primer orden, o primarios. El Cuadro 2 muestra la relación entre la primera y la segunda orden, así como los asuntos del BIS-11 en los factores. 
Cuadro 2 - Factores primarios y secundarios de la BIS 11.

\begin{tabular}{|c|c|c|c|c|}
\hline \multicolumn{5}{|c|}{ Escala de Impulsividad de Barratt - BIS 11} \\
\hline $\begin{array}{c}\text { Factores } \\
\text { secundarios }\end{array}$ & & $\begin{array}{l}\text { Factores } \\
\text { primarios }\end{array}$ & $\begin{array}{l}\text { No de } \\
\text { ítems }\end{array}$ & $\begin{array}{l}\text { Ítems que contribuyen para } \\
\text { cada subescala }\end{array}$ \\
\hline \multirow{2}{*}{$\begin{array}{l}\text { Impulsividad } \\
\text { Atencional }\end{array}$} & \multirow{2}{*}{$\begin{array}{l}\text { Impulsividad relacionada a toma de } \\
\text { decisión rápida }\end{array}$} & Atención & 5 & $5,9^{*}, 11,20^{*}, 28$ \\
\hline & & $\begin{array}{c}\text { Inestabilidad } \\
\text { cognitiva }\end{array}$ & 3 & $6,24,26$ \\
\hline \multirow{2}{*}{$\begin{array}{l}\text { Impulsividad } \\
\text { Motora }\end{array}$} & \multirow{2}{*}{$\begin{array}{c}\text { La impulsividad motora está relacionada } \\
\text { a la no inhibición de respuestas } \\
\text { incoherentes con el contexto }\end{array}$} & Motor & 7 & $2,3,4,17,19,22,25$ \\
\hline & & Perseverancia & 4 & $16,21,23,30^{*}$ \\
\hline \multirow{2}{*}{$\begin{array}{l}\text { Impulsividad por } \\
\text { no planificación }\end{array}$} & \multirow{2}{*}{$\begin{array}{c}\text { Engloba comportamientos orientados al } \\
\text { presente }\end{array}$} & Autocontrol & 6 & $1^{*}, 7^{*}, 8^{*}, 12^{*}, 13^{*}, 14$ \\
\hline & & $\begin{array}{c}\text { Complejidad } \\
\text { cognitiva }\end{array}$ & 5 & $10^{*}, 15^{*}, 18,27,29 *$ \\
\hline & & & & $*$ análisis inverso $(4,3,2,1$ \\
\hline
\end{tabular}

Fuente: El autor.

Mientras muchos manuscritos académicos relatan solamente la puntuación total, es recomendado que, por lo menos, se relate el segundo factor de orden para explicar su contribución individual para la relación que se está testando (PATTON et al., 1995).

Los resultados pueden variar de 30 (treinta) a 120 (ciento veinte) puntos. Para el caso de 30 (treinta) puntos, el encuestado que, en las preguntas normales, opte por la alternativa "raramente o nunca" $\mathrm{y}$, en las cuestiones invertidas, opte por la alternativa "casi siempre/siempre", recibirá 1 (un) punto por asunto. Para el caso de 120 (ciento veinte) puntos, el encuestado que opte por la alternativa inversa al caso anterior recibirá, así, 4 (cuatro) puntos por pregunta.

Para el análisis de la impulsividad, los resultados que consideran los menos impulsivos son los que se aproximaron a 30 (treinta) y los más impulsivos, aquellos que se aproximaron a 120 (ciento veinte) puntos. En esta investigación, fueron considerados menos impulsivos los voluntarios con puntuación en el primer cuartil y, más impulsivos, los situados en el tercer cuartil, o sea, los extremos de la muestra.

En una segunda etapa de la investigación, se formatearon los datos con la inclusión de la puntuación obtenida en la prueba TAPMIL y las notas del Escuadrón de Instrucción Aérea (EIA), lugar donde los cadetes reciben la instrucción práctica de vuelo. Es importante recordar que la prueba TAPMIL evalúa seis factores, habiéndose considerado, para esta investigación, solo el factor capacidad para la ejecución de múltiples tareas.

Para lograr las informaciones fueron realizados análisis descriptivos (promedio, desvío estándar, varianza, máximo, mínimo, amplitud, entre otras). Para complementar, se verificó el coeficiente de variación de las variables en estudio.

Con los datos agrupados en pares fue verificado el coeficiente de correlación.

A continuación, en el tópico 4, tablas 1 a 7 , son presentados los resultados.

\section{RESULTADOS}

4.1 Análisis descriptivo de las pruebas TMT y BIS

Tabla 1 - TMT.

\begin{tabular}{cc} 
& TMT \\
\hline Promedio & 93,53333 \\
Error estándar & 5,495232 \\
Mediana & 88 \\
Moda & 136 \\
Desvío estándar & 36,86314 \\
Varianza de la muestra & 1358,891 \\
Curtosis & 0,162757 \\
Asimetría & 0,73699 \\
Intervalo & 158 \\
Mínimo & 40 \\
Máximo & 198 \\
Suma & 4209 \\
Conteo & 45 \\
\hline
\end{tabular}

Fuente: El autor. 
Tabla 2 - BIS.

\begin{tabular}{cc}
\hline & BIS \\
\hline Promedio & 61,26667 \\
Error estándar & 1,664362 \\
Mediana & 61 \\
Moda & 66 \\
Desvío estándar & 11,16488 \\
Varianza de la muestra & 124,6545 \\
Curtosis & $-0,53319$ \\
Asimetría & 0,236712 \\
Intervalo & 45 \\
Mínimo & 42 \\
Máximo & 87 \\
Suma & 2757 \\
Conteo & 45 \\
\hline
\end{tabular}

Fuente: El autor.

El coeficiente de variación del BIS es del 18,22\% y del TMT del 39,41\%, lo que significa que, abajo del $30 \%$, la muestra es considerada homogénea y, por encima de ese porcentaje, considerada heterogéneo.

Tabla 3 - TAPMIL.

\begin{tabular}{cc}
\hline \multicolumn{1}{c}{ TAPMIL } \\
\hline Promedio & 111,0667 \\
Error estándar & 2,490061 \\
Mediana & 109 \\
Moda & 98 \\
Desvío estándar & 16,70384 \\
Varianza de la muestra & 279,0182 \\
Curtosis & $-0,00887$ \\
Asimetría & $-0,12804$ \\
Intervalo & 82 \\
Mínimo & 64 \\
Máximo & 146 \\
Suma & 4998 \\
Conteo & 45 \\
\hline
\end{tabular}

Fuente: El autor.
Tabla 4 - EIA.

\begin{tabular}{cc}
\hline & EIA \\
\hline Promedio & 4,374933 \\
Error estándar & 0,029532 \\
Mediana & 4,343 \\
Moda & 4,257 \\
Desvío estándar & 0,198105 \\
Varianza de la muestra & 0,039245 \\
Curtosis & $-0,42877$ \\
Asimetría & 0,516116 \\
Intervalo & 0,8 \\
Mínimo & 4,029 \\
Máximo & 4,829 \\
Suma & 196,872 \\
Conteo & 45
\end{tabular}

Fuente: El autor.

El coeficiente de variación del TAPMIL es del $15,04 \%$ y del EIA, de del 4,53\%, ambos abajo del $30 \%$, lo que significa homogeneidad de los datos.

\subsection{Coeficiente de correlación}

En este subtópico son presentados los coeficientes de las pruebas TMT, TAPMIL y la nota del EIA en relación a la prueba BIS (impulsividad).

Tabla 5 - Correlación 1.

\begin{tabular}{ccc}
\hline & Correlación 1 \\
\hline BIS & BIS & TMT \\
TMT & 1 & 1 \\
\hline
\end{tabular}

Fuente: El autor.

Tabla 6 - Correlación 2.

\begin{tabular}{ccc}
\hline & Correlación 2 \\
\hline BIS & BIS & TAPMIL \\
TAPMIL & 1 & 1 \\
\hline Fuente: El autor & $-0,023982923$ &
\end{tabular}

Tabla 7 - Correlación 3.

\begin{tabular}{ccc}
\hline & Correlación 3 \\
\hline BIS & BIS & EIA \\
EIA & 1 & 1 \\
\hline
\end{tabular}

Fuente: El autor. 
Se verifica que no hay correlación de la impulsividad con las otras pruebas y, también, que su relación es inversa. O sea, la conclusión a que se llega con el análisis de los datos de las pruebas TMT, BIS 11, TAPMIL y notas en el EIA es de que los cadetes más hábiles en administrar múltiples tareas, así como los más hábiles en vuelo, no son aquellos que presentan la mayor impulsividad.

Esa conclusión es bastante propicia, pues la impulsividad es una característica bastante indeseable en el medio militar, sobre todo tratándose de la aviación militar.

\section{CONSIDERACIONES FINALES}

El presente estudio partió del interés de verificar si la variable impulsividad interfiere en la ejecución de múltiples tareas y si la misma afecta el desempeño en vuelo. La investigación de Araújo et al. (2009) concluyó que la impulsividad está asociada al comportamiento de riesgo en el tránsito, correr por aventura e infracciones.

De lo que fue propuesto para este trabajo, no hubo correlación entre la variable impulsividad y la ejecución de múltiples tareas, tampoco asociación entre la impulsividad y el desempeño en vuelo.

\section{REFERENCIAS}

ARAÚJO, M. M.; MALLOY-DINIZ, L. F.; ROCHA, F. L. Impulsividade e acidentes de trânsito. Revista de Psiquiatria Clínica, v. 36, n. 2, p. 60-68, 2009.

BAZERMAN, M. H. Judgment in managerial decision making. 6th ed. Hoboken: J. Wiley \& Sons, 2006.

\section{COSTA, M. P. Estudo de normatização e} validação do teste de aptidão para a pilotagem militar. 2010. Dissertação (Mestrado) - Instituto de Psicologia, Universidade de São Paulo, 2010.

DAVEL, E.; VERGARA, S. C. (Org.). Gestão com pessoas e subjetividade. São Paulo: Atlas, 2001.

FÁVERO, L. P. et al. Análise de Dados: modelagem multivariada para tomada de decisões. Rio de Janeiro: Elsevier, 2009.

KLEIN, G. Sources of power: how people make decisions. Cambridge: MIT, 2000.

MALLOY-DINIZ, L. F. et al. Impulsiveness scale (BIS-11) para aplicação em adultos brasileiros.
Jornal Brasileiro de Psiquiatria, Rio de Janeiro, v. 59, n. 2, p. 99-105, 2010.

HERCULANO-HOUZEL, S. O cérebro nosso de cada dia. Rio de Janeiro: FAPERJ: CNPQ, 2000. Disponível em:< http://www.cerebronosso.bio.br/guiabsico-de-neurocincia>. Acesso em: 5 mar 2014.

PATTON, J. H.; STANFORD, M. S.; BARRATT, E.S. BIS-11 instrument reference. Factor structure of the Barratt impulsiveness scale. Journal of Clinical Psychology, v. 51, n. 6, p. 768-774. Disponível em: <http://www.impulsivity.org/measurement/bis11>. Acesso em: 25 mar. 2014.

SANBONMATSU, D. M et al. Who multi-tasks and why? Multi-tasking ability, perceived multi-tasking ability, impulsivity, and sensation seeking. PSO ONE, v. 8, n. 1, jan. 2013.

SILVA, L. M. A.; LUCAS, L. A.; COSTA, M. P. Analysis of decision making processo flight instructors at the Brazilian Air Force Academy. In: INTERNATIONAL CONFERENCE ON NATURALISTIC DECISION MAKING, 9., 2009, London. Proceedings of ... London: [s .n.], 2009. 